\title{
Detección de trombos intracardíacos: buscando la mejor técnica
}

El enfrentamiento de las masas intracardíacas ha sido siempre desafiante en la practica clínica. Basado en la experiencia de la literatura sabemos que la mayor parte de las masas dentro de las cavidades cardíacas corresponden a trombos, seguidos por tumores tipo mixoma y posteriormente por fibroelastomas. Por mucho tiempo la ecocardiografía ha sido el método de diagnóstico y evaluación de estas masas intracardíacas. Las ventajas de portabilidad, costo y ubicuidad la han llevado a ser la técnica de imagen mas solicitada ${ }^{1}$. Sin embargo la introducción en la ultima década de la resonancia magnética cardíaca (RMC) ha desafiado este algoritmo ${ }^{2}$. Mas allá de las ventajas de la RMC por su resolución espacial y temporal, es la posibilidad de tener un acercamiento de la composición de la masa lo que la hace una técnica muy atractiva ${ }^{3}$. Además, la capacidad de visualizar las estructuras extracardíacas es de utilidad en la practica en especial cuando se sospecha de procesos tumorales expansivos.

En la presente edición de la Revista, el Dr. Julián Noche y co-autores, describen los pacientes con hallazgo de trombo en la RMC que tenían previamente estudio ecocardio- gráfico transtoracico normal. En un estudio descriptivo y usando una base de datos extranjera, los autores revisan una serie de pacientes referidos a estudio RMC por diversos diagnósticos. Cabe destacar que en los pacientes no había sospecha de trombo dentro de los diagnósticos de referencia. Los autores concluyen que la RMC es una técnica muy superior a la ecocardiografía en la detección de trombos y debiera considerarse dentro del algoritmo de estudio de fuente embolígenas.

En el análisis del artículo hay que considerar que no se trata de un estudio directamente comparativo uno a uno, sino que es una descripción retrospectiva basada en los hallazgos de la RMC. Además, existe una ventana de tiempo (hasta 3 meses) en los cuales la situación del paciente pudo haber cambiado (no hay información del tratamiento concomitante). Dentro del análisis destaca la no inclusión del la orejuela izquierda, fuente habitual de trombos en especial en pacientes con fibrilación auricular. Es justamente en esta última localización donde la RMC pudiera darnos falsos negativos, es sabido que por las diferencias de resolución temporal entre ambas técnicas, masas muy peque-

Correspondencia: 
ñas pudieran no ser visualizadas por la resonancia.

Sin embargo, a pesar de las limitaciones propias de un estudio descriptivo, los autores nos llevan a plantear cual es la mejor técnica de evaluación en pacientes en estudio de fuente embólica. Esto es muy importante al momento de definir pautas de estudio de los pacientes, las cuales muchas veces consideran solo el costo como un factor determinante y no cuál es el mejor método y el más completo desde la perspectiva del paciente. Desde la perspectiva clínica, además, otras interrogantes surgen de este traba- jo, por ejemplo, cuál es el mejor método de seguimiento para un paciente con diagnóstico de trombo, dado que la suspensión del tratamiento anticoagulante depende de ello es necesario contar con una técnica confiable para tomar esas decisión. En el medio nacional se ha progresado en la disponibilidad de métodos de imagen en diversos centros, por lo cual experiencias multicentro son más factibles que en el pasado para contestar interrogantes clínicas. En este sentido el presente artículo debe ser considerado como un estímulo para estudios en al ambiente nacional que nos lleven a responder ésta y otras preguntas.

\section{Referencias:}

1. ACCF/ASE/AHA/ASNC/HFSA/HRS/SCAI/SCCM/SCCT/ SCMR 2011 Appropriate Use Criteria for Echocardiography. Journal of the American Society of Echocardiography. 2011; 24: $229-267$

2. SRICHAI MB1, JUNOR C, RODRIGUEZ LL, STILLMAN AE, GRIMM RA, LIEBER ML, et al. Clinical, imaging, and pathologic characteristics of left ventricular thrombus: a comparison of contrast enhanced magnetic resonance imaging, transthoracic echocardiography and transesophageal echocardiography with surgical or pathological validation. Am Heart J 2006; 152: 75-84.

3. GULATI G, SHARMA S, KOTHARI SS, JUNEJA R, SAXENA A, TALWAR KK. Comparision of Echo and MRI in the Imaging Evaluation of Intracardiac Masses. Cardiovas Interventional Radiol 2004; 27: 459-469. 